\title{
Plantar Pressure Changes and Their Relationships with Low Back Pain during Pregnancy Using Instrumented Insoles
}

\author{
Fernando Martínez-Martí, ${ }^{1}$ Olga Ocón-Hernández, ${ }^{2}$ María Sofía Martínez-García, ${ }^{3}$ \\ Francisco Torres-Ruiz, ${ }^{4}$ Antonio Martínez-Olmos ${ }^{(D},{ }^{1}$ Miguel A. Carvajal, ${ }^{1}$ Jesús Banqueri, ${ }^{1}$ \\ and Alberto J. Palma ${ }^{1}{ }^{1}$ \\ ${ }^{1}$ ECsens, CITIC-UGR, Departamento de Electrónica y Tecnología de Computadores, ETSIIT, Universidad de Granada, \\ 18071 Granada, Spain \\ ${ }^{2}$ Servicio de Obstetricia y Ginecología, Hospital Universitario San Cecilio, 18071 Granada, Spain \\ ${ }^{3}$ HCTLab, Departamento de Tecnología Electrónica y las Comunicaciones, Universidad Autónoma de Madrid, 28049 Madrid, Spain \\ ${ }^{4}$ Departamento de Estadística e Investigación Operativa, Facultad de Ciencias, Universidad de Granada, 18071 Granada, Spain
}

Correspondence should be addressed to Alberto J. Palma; ajpalma@ugr.es

Received 26 September 2018; Accepted 23 December 2018; Published 24 February 2019

Academic Editor: Carlos Marques

Copyright (C) 2019 Fernando Martínez-Martí et al. This is an open access article distributed under the Creative Commons Attribution License, which permits unrestricted use, distribution, and reproduction in any medium, provided the original work is properly cited.

Low back pain affects around 50\% of pregnant women and presents significant morbidity and persistence for years in $20 \%$ of postpartum women who report that pain. Numerous studies have documented gait alterations during pregnancy and postpartum. Therefore, an analysis of the relationship between certain gait parameters and low back pain was attempted using low-cost validated instrumented insoles. This work presents a longitudinal cohort study carried out during routine gynecological follow-up visits in the first, second, and third trimesters of pregnancy at an Obstetrics and Gynecology Service. Sample size was 62. Plantar pressure data were collected with specially designed instrumented insoles containing four force sensors to measure peak pressure, center of pressure, and stance phase time in each foot and in each pregnancy trimester. The analysis was carried out on a two-dimensional level, simultaneously considering the data from both feet using Hotelling's $T^{2}$ test. This longitudinal study detected relationships between certain gait parameter changes and low back pain during pregnancy. It revealed a cyclic tendency of low back pain prevalence with a maximum in the second trimester and a decrease in the third trimester, which was correlated with alterations of the pregnant gait: excessive foot pronation and rearfoot pressure increase.

\section{Introduction}

Pregnancy has profound physiological effects on a woman's body, affecting not only the cardiovascular, endocrine, and renal systems but also the musculoskeletal system, with altered gait and posture characteristics. These gait and posture changes could lead to compensatory mechanisms that may overload body segments and cause discomfort and pain $[1,2]$. Indeed, low back pain (LBP) is commonly experienced during pregnancy, affecting approximately $50 \%$ of pregnant women [3]. It is associated with significant morbidity in pregnant and postpartum women, with persistent pain for several years in some patients [4-6]. The etiology of LBP is still unclear [7]; it is considered to be the natural course of pregnancy and is expected to disappear spontaneously after delivery. Further, it is the result of quite a few factors, such as mechanical, hormonal, and other aspects [8].

Previous research into the gait biomechanics of pregnant women referred to kinetic variables, such as ground reaction forces [2, 9], kinematics [2, 10-13], spatiotemporal parameters $[9,14-19]$, and plantar pressure distribution $[13,17$, 19-25]. More literature about LBP, posture, and gait changes during pregnancy has been assessed in some interesting reviews [26, 27]. Researching dynamic plantar pressure distribution, for instance, Nyska et al. found higher rearfoot pressures and contact times in last-trimester pregnant 
women compared to a nonpregnant group [20]. Similar results were observed in a longitudinal study throughout the pregnancy, with increased pressures in the rearfoot and reduced pressures in the forefoot in the last trimester [21]. Likewise, a significant increase in midfoot plantar pressure was also observed at 38 weeks of gestation compared to controls [28]. Nevertheless, although larger contact areas and longer times were confirmed, some authors observed reduced plantar load in the rearfoot with increased pressure in the midfoot and forefoot during pregnancy $[14,22]$ or even minimal force parameter changes [23] with some mediolateral deviations of the foot center of pressure (CoP) [17, 19]. Moreover, an altered mean pressure distribution and CoP trajectory was observed throughout the pregnancy compared with postpartum [13].

There are few studies analyzing the correlation between posture or gait features and pregnancy-related pain (low back, pelvic, hip, lower limb, and foot pain). No significant correlation was observed between LBP and lumbar lordosis $[1,29]$ or lower limb pain and pronated foot posture [30]. A weak correlation was also found between foot pain and forefoot contact time but no correlation with plantar pressure [14], whereas higher forces in the midfoot and a broader contact area were correlated to the presence and severity of foot pain [25]. Reported results can be used to understand the musculoskeletal dysfunctions of pregnant women to design exercises for the preventive and rehabilitative treatment of pain $[31,32]$.

In-shoe systems can be used to record plantar-pressure distributions with a sensorized insole within a shoe. Commercial products include the F-scan measurement system (Tekscan Inc., South Boston, USA) and the Novel Pedar system (Novel USA Inc.), all of which capture dynamic in-shoe temporal and spatial pressure distributions that are used for multiple gait and analysis. However, in most cases, these systems are conceived for indoor or laboratory use. On the other hand, OpenGo Science (Moticon, Munich, Germany) is another commercial solution which is a wireless and flexible way to analyze the plantar pressure distribution, contact forces, and dynamics of the human foot. Several research groups have also developed their own sensorized insoles for multiple applications [33]. One of the first plantar measurement systems was designed by Zhu et al. [34] in 1990 with the goal of measuring the pressure distribution beneath the foot to distinguish pressure during walking and shuffling. This system contained seven force-sensitive resistors (FSR, Interlink, USA). Regarding more recent and wireless instrumented insoles, a wireless insole, based on custom capacitive technology, was used to monitor the timing and movement of the athletes' legs during throwing, jumping, and running in several sport disciplines [35]. Shu et al. [36] developed an in-shoe plantar pressure measurement and analysis system based on fabric pressure sensing array. The pressure sensors were fabricated using a conductive sensing fabric with conductive yarns and a top-and-bottom conversion layer. Moreover, a novel and very recent system was based on optical fiber sensors for plantar pressure measurements [37].

Therefore, with the aim of a better understanding of the relationship of LBP and gait, a longitudinal study was carried out using our developed in-shoe system to analyze plantar pressure distributions and the stance phase time of a group of healthy pregnant women throughout their pregnancy in the first (T1), second (T2), and third (T3) trimesters. Our preliminary results with a smaller group and a different statistical analysis were already presented showing the relationship between the anteroposterior shifted center of pressure and the LBP in the third trimester [38].

\section{Materials and Methods}

2.1. Participants. Seventy pregnant women who attended their first prenatal visit at the Unit of Obstetrics and Gynecology at the University Hospital San Cecilio of Granada (Granada, Spain) started this longitudinal study, and sixty-two $(88.6 \%)$ finished it. The eight pregnant women (11.4\%) who did not finish the study had complications or special care needs during pregnancy. Women with a medical record of back, foot, ankle, knee, musculoskeletal, or neuromuscular trauma or disease as well as women pregnant with twins were discarded as exclusion criteria. All participants received full information about the study and gave informed consent. This study was previously approved by the Clinical Research Ethics Committee of Granada, Government of Andalusia, Spain.

2.2. Instrumented Insoles. A low-cost instrumented insole system designed by our research group was used (Figure 1) for the dynamic pressure measurements. Our system was previously validated as a pressure-measuring instrument [39] using a commercial system, F-Scan (Tekscan, South Boston, MA, USA) and as a time-measuring system of physical activity in comparison with two gold standards [40]. With the aim of simplicity, each insole comprised a flexible polymeric film supporting a total of four piezoresistive pressure sensors located at the heads of the first and fifth metatarsals, at the first toe, and at the heel. The FlexiForce A201 sensors were used (Tekscan, USA) which is a piezoresistive sensor connected with a planar flexible wire whose sensing area is a circle with a diameter of $9.53 \mathrm{~mm}$ registering pressures of up to $1500 \mathrm{kPa}$. Sensor reliability was tested by using them for around $100 \mathrm{~km}$ in our combined tests, without damage or appreciable aging in any of the eight sensors. Each sensor was individually calibrated in the range of interest (up to $400 \mathrm{kPa}$ for the metatarsal pressure sensors and up to $1000 \mathrm{kPa}$ for the heel and toe sensors) with a press, obtaining a nonlinearity and hysteresis total error of less than $5 \%$. The pressure and conductance of the sensors were fitted to linear regression, and the coefficients were used to calculate the pressure.

An insole thickness of $1 \mathrm{~mm}$ allows them to be used in regular, unmodified shoes and therefore was not expected to influence the standard gait tests. Pressure data from each sensor were collected with a sampling frequency of $40 \mathrm{~Hz}$. In addition, the information collected from the sensors was processed in a datalogger fastened at the waist. More technical details of the instrumented insole system can be read elsewhere $[39,40]$. 

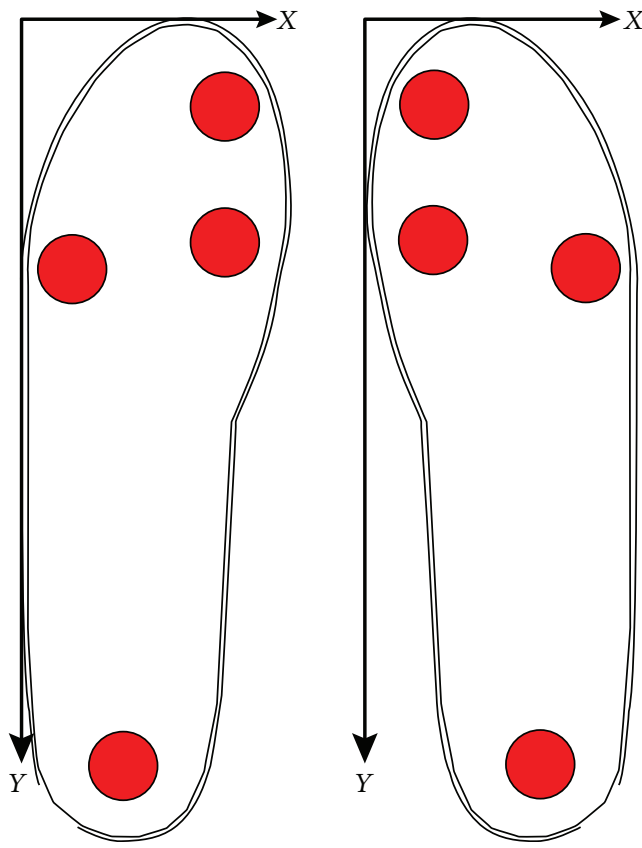

(a)

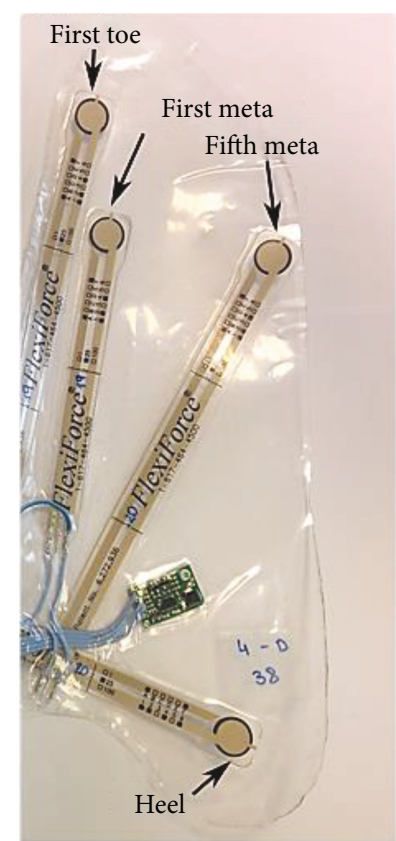

(b)

FIgURE 1: Sketch of the instrumented insoles showing the sensor positions and the $X-Y$ coordinate axis (a). Picture of the right insole (b). The diameter of each sensor was $9.53 \mathrm{~mm}$.

2.3. Data Collection. Dynamic pressure data were collected during the routine prenatal visits of each participant in the first trimester (T1), second trimester (T2), and third trimester (T3) (around the 12th, 20th, and 32nd weeks of pregnancy, respectively). In the three visits, participants were asked to rate their perceived low back pain with a numeric rating scale (NRS) from 0 to 9 (where 0 represents no pain at all and 9 is the worst pain imaginable) [41]. Then, the subjects were binarily categorized: those who rated it 0 were categorized in the no-pain group and the rest in the pain group [25, 30, 42]. After that, participants were provided with the same model of sports shoes with the instrumented insoles included. Different sizes of shoes and insoles were available to accommodate each subject. Participants were instructed to walk freely to adapt to the shoes with the insoles and environment before gait assessment. The gait test was carried out walking at a preferred participant speed in a hospital corridor 10 meters in length (back and forth twice). The initial and final steps were discarded to reduce boundary condition influence (acceleration and deceleration steps) on the experiment, and a mean of 40 steps was used for statistical analysis.

2.4. Data Analysis. The $X-Y$ coordinate origins were at the left front corner of each foot, with the $X$-axis in the mediolateral direction and the $Y$-axis in the anteroposterior direction (Figure 1). The CoP and the stance phase time of each foot can be evaluated with the instrumented insole. Therefore, we selected the following gait parameters:

(1) The time-integrated planar coordinates of the dynamic $\mathrm{CoP}$ of each foot, $\mathrm{XCoP}$, and $Y \mathrm{CoP}$ were calculated by implementing a time integration used by the commercial instrumented insoles F-Scan [43]:

$$
\begin{aligned}
& X \mathrm{CoP}=\frac{\sum_{i=1}^{4}<P_{i}^{\alpha}>\cdot x_{i}^{\alpha}}{\sum_{i=1}^{4}<P_{i}^{\alpha}>}, \\
& Y \mathrm{CoP}=\frac{\sum_{i=1}^{4}<P_{i}^{\alpha}>\cdot y_{i}^{\alpha}}{\sum_{i=1}^{4}<P_{i}^{\alpha}>},
\end{aligned}
$$

where $\alpha$ refers to the left (L) or right (R) foot, $X_{i}$ and $Y_{i}$ are the cartesian coordinates which are invariable for each single sensor, and $\left\langle P_{i}\right\rangle$ is the timeintegrated pressure on that sensor throughout the contact time. This feature is used to show how the plantar pressures are balanced during each stance phase over the entire foot

(2) The peak pressure average on valid steps of the first toe and heel sensors to study the anteroposterior trend or forefoot/rearfoot peak pressure balance

(3) The peak pressure average on valid steps of the 1st and 5th metatarsals to study the mediolateral pressure balance or supination/pronation trend

(4) The stance phase time of every single foot

In Figure 2, typical pressure data from the four sensors of one participant's foot are displayed as a function of time showing the four pressure peaks considered and how the stance phase time was calculated. Although not considered in this study, the swing (noncontact) phase is also shown in Figure 2. These gait parameter differences between 


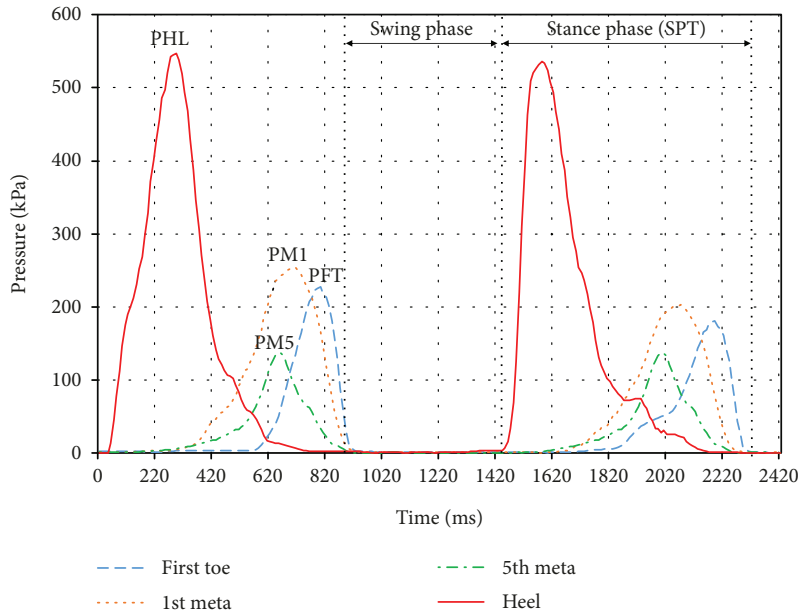

FIGURE 2: Typical pressure data from the four sensors showing the gait parameters considered in this study during an actual test. PHL is the peak pressure at the heel sensor, PM1 and PM5 are the peak pressures at the first and fifth metatarsal heads, and PFT is the peak pressure at the first toe. Stance phase time was calculated by measuring the time from when the heel strikes until the toe-off.

consecutive visits were considered in this study and termed as shown in Table 1.

In accordance with LBP categorization, a binary notation was used for the pain rating. Thus, no LBP was noted by "0" and LBP by " 1 ". Consequently, the LBP transitions between visits were 00 (no LBP/no LPB), 01 (no LBP/LBP), 10 (LBP/no LBP), and 11 (LBP/LBP), where the first digit corresponds to the first trimester.

2.5. Statistical Study and Study Definition. First, an analysis of the normality variable was carried out using the Shapiro-Wilks statistic, as well as the detection of anomalous and/or influential data by means of the Grubb test. For each of the above-mentioned gait parameters, significant differences were found between the mean values of the parameters between two consecutive trimesters. The analysis was carried out at the two-dimensional level, simultaneously considering the data from both feet using Hotelling's $T^{2}$ test. When significant differences $(p<0.05)$ were found, the $95 \%$ Bonferroni simultaneous intervals for the mean differences were calculated between consecutive visits.

In each case, and following this methodology, the analysis followed three successive phases:

Study A: Differences in plantar pressure and stance phase time between consecutive trimesters

Study B: The analysis in study A was categorized according to the presence of pain in the last of the follow-up visits

Study C: Differences were also studied considering the pain transitions between consecutive followup visits
TABLE 1: Gait parameter average for all valid steps.

\begin{tabular}{lccc}
\hline Weeks & Foot & Parameter & Parameter description \\
\hline $\begin{array}{l}\text { T2_1: differences } \\
\text { between T2 and T1 }\end{array}$ & R: right & YCoP & $\begin{array}{c}\text { Center of pressure } \\
\text { (X-coordinate } \\
\text { Center of pressure } \\
\text { (Y-coordinate) } \\
\text { Peak pressure at the } \\
\text { first toe sensor } \\
\text { Peak pressure at the } \\
\text { heel sensor }\end{array}$ \\
\hline $\begin{array}{l}\text { T3_2: differences } \\
\text { between T3 and T2 }\end{array}$ & L: Left & PM5 & $\begin{array}{c}\text { Peak pressure at the } \\
\text { 5th metatarsal sensor } \\
\text { Stance phase time }\end{array}$ \\
\hline
\end{tabular}

\section{Results}

Anthropometric parameters of participants throughout the pregnancy are shown in Table 2.

3.1. Perceived Pain Categorization. Binary pain-rating data are displayed in Table 3 for each follow-up visit.

The number of participants involved in the different transitions between presence (1) or absence of LBP (0) between $\mathrm{T} 1$ and $\mathrm{T} 2$ is shown in Table 4 . Table 5 shows the same information from $\mathrm{T} 2$ to $\mathrm{T} 3$.

From Tables 3-5, a notable increase in the perceived pain from $\mathrm{T} 1$ to $\mathrm{T} 2$ was mentioned and was then partially reversed in T3. Therefore, there were a significant number of subjects who changed from no pain to pain (01) in T2 (18), while the number of subjects who kept perceiving no pain remained almost stable (00: 20-18) in the last two trimesters. Consequently, there were very few participants in the pain transitions 10 (T2) and 01 (T3), and therefore, the analysis of these cases may have scant significance.

3.2. Study A: Gait Parameter Changes throughout Pregnancy. Table 6 includes the $95 \%$ confidence intervals for the statistically significant differences between consecutive follow-up visits, with $X \mathrm{CoP}$ and $Y \mathrm{CoP}$ given in $\mathrm{cm}$; PFT, PHL, PM1, and PM5 in $\mathrm{kPa}$; and SPT in milliseconds. Graphic representations of parameter trends are also shown in Table 6, where triangles and inverted triangles indicate an increase and decrease in peak pressures between visits, respectively, and arrows point out the shifted CoP direction. A PHL increase and SPT decrease in both feet, with a PM1 and PM5 increase in the left foot, and a medially shifted $\mathrm{CoP}$ in the right foot, were observed in T2_1. In T3_2, the CoP becomes anteriorly shifted (right foot), the PHL is reduced in the left foot, and PM1 increases in both feet together with a PM5 increase only in the right foot.

3.3. Study B: Gait Parameter Changes Categorized by Perceived LBP throughout the Pregnancy. The previous results were categorized by perceived pain in the last trimester in each case (Table 7). In the case of no LBP (0), we 
TABle 2: Anthropometric parameters across the study $(n=62)$.

\begin{tabular}{lcc}
\hline & Mean & Std. deviation \\
\hline Age (years) & 31.42 & 4.44 \\
Height $(\mathrm{cm})$ & 163.47 & 7.52 \\
Weight T1 $(\mathrm{kg})$ & 63.83 & 11.72 \\
Weight T2 $(\mathrm{kg})$ & 69.99 & 11.72 \\
Weight T3 $(\mathrm{kg})$ & 76.01 & 12.75 \\
\hline
\end{tabular}

TABLE 3: Number and percentage of participants categorized by LBP rate and pregnancy trimester.

\begin{tabular}{lllllll}
\hline Trimester & \multicolumn{2}{c}{ T1 } & \multicolumn{2}{c}{ T2 } & \multicolumn{2}{c}{ T3 } \\
No pain (0) & 38 & $61.3 \%$ & 23 & $37.1 \%$ & 28 & $45.2 \%$ \\
\hline Pain (1) & 24 & $38.7 \%$ & 39 & $62.9 \%$ & 34 & $54.8 \%$ \\
Total & 62 & $100.0 \%$ & 62 & $100.0 \%$ & 62 & $100.0 \%$ \\
\hline
\end{tabular}

TABLE 4: Number of participants categorized by LBP transition from $\mathrm{T} 1$ to $\mathrm{T} 2$.

\begin{tabular}{lcccc}
\hline & & \multicolumn{2}{c}{ T2 } & Total \\
& & No pain $(0)$ & Pain $(1)$ & \\
\hline \multirow{2}{*}{ T1 } & No pain (0) & 20 & 18 & 38 \\
& Pain (1) & 3 & 21 & 24 \\
\hline Total & & 23 & 39 & 62 \\
\hline
\end{tabular}

TABLE 5: Number of participants categorized by LBP transition from $\mathrm{T} 2$ to $\mathrm{T} 3$.

\begin{tabular}{lcccc}
\hline & & \multicolumn{2}{c}{ T3 } & Total \\
& & No pain $(0)$ & Pain $(1)$ & \\
\hline \multirow{2}{*}{ T2 } & No pain (0) & 18 & 5 & 23 \\
& Pain (1) & 10 & 29 & 39 \\
\hline Total & & 28 & 34 & 62 \\
\hline
\end{tabular}

observed an antero-shifted CoP and PM1 increase in the left foot together with SPT reduction in both feet in T2 with respect to T1. From T2 to T3, a laterally shifted CoP and PHL decrease was also measured in the left foot although no significant change was observed in SPT. In the case of LBP (1), PHL increases and an anteriorly shifted CoP were observed in the right foot, together with a PM1 increase in the right foot from T1 to T2. Studying T3_2 with LBP, we observed an anteriorly shifted CoP and PM1 and PHL increases in the left foot and a PM5 increase in the right foot.

3.4. Study C: Gait Parameter Changes Categorized by Perceived LBP Transitions between Consecutive Trimesters. Table 8 presents the categorization of Table 7 data by the aforementioned LBP transitions between consecutive trimesters. Two of the eight LBP transitions did not show any significant correlations (T2_1 10 and T3_2 01), coinciding with transitions involving very few participants. For the rest of the cases, from T1 to T2, a shifted CoP to the forefoot in both feet was correlated with no LBP in T2. A medially shifted CoP in both feet (LBP transition: 01) or in the right foot (LBP transition: 11), along with a PHL increase in both feet or the right foot, was correlated with LBP in T2. From T2 to T3, an anterior and laterally shifted CoP with a PHL decrease was observed in the left foot in patients with no LBP in both the last trimesters (LBP transition: 00). In transition 11, no lateral shift of the CoP and a PM1 increase were observed in comparison with the previous case. A forefoot-shifted CoP was observed for participants who changed their LBP perception from pain in T2 to no pain in T3 (LBP transition: 10).

\section{Discussion and Conclusions}

This longitudinal study is aimed at assessing the relation between dynamic plantar pressure and stance phase time changes and LBP evolution throughout pregnancy. Our results show high LBP prevalence in $\mathrm{T} 1$, as observed in a prior study [4], with a notable increase in perceived pain in the second trimester (62.9\%), similar to previous reports $[44,45]$, with a slight decrease in T3 (54.8\%) in agreement with data from the untreated group of the George et al. report [46]. This is also in agreement with the review by $\mathrm{Wu}$ et al. with prevalence peaks between 24 and 36 weeks [6]. At any rate, pain prevalence from this study coincides with previous findings in the literature, where the average prevalence published was $45.3 \%$, with large variations (range $3.9-89.9 \%)[6,27]$.

From Tables 6,7 , and 8 , a medially shifted CoP or pronating tendency was observed, as in most static and dynamic published studies [20, 23, 30, 47-52]. In a recent report, pronation was also observed without correlation with pain in the lower limbs [30]. Our data show that pronation was more pronounced in the right foot and mostly correlated with perceived LBP. Indeed, a symmetric pronated trend in both feet was correlated with new perceived LBP in T2 (01 in Table 8). Pronation of the foot results in internal rotation of the tibia caused by the increased calcaneal eversion angle, which can lead to discomfort and back pain in some pregnant women $[52,53]$. On the other hand, a shift of the CoP towards the forefoot $(Y \mathrm{CoP}<0)$ was also observed in the last two trimesters, but only related to the painless condition with an absence of peak pressure increases (transitions 00 in T2_1 and T3_2). This CoP shift towards the forefoot has been previously reported [26].

Moreover, lateralization in the left foot $\mathrm{CoP}$ in $\mathrm{T} 3$ $(X \mathrm{CoP}>0)$ is always correlated with no LBP, 0 , in study B (Table 7), and the continued no-pain condition, 00 , in study C (Table 8), in agreement with previous studies $[13,17,20$, 24]. This laterally shifted CoP has been attributed to better dispersing increasing body weight. In this regard, some studies that did not observe a shifted CoP suggested the use of extra muscle work to explain an unchanged gait pattern in pregnant women $[2,23]$. This overuse of the musculoskeletal system may lead to disorders of several body segments such as LBP [2], among others [54].

Except for the first toe (with no significant correlation observed), peak pressure results confirmed the time- 
TABLE 6: Study A: gait parameter changes throughout pregnancy.

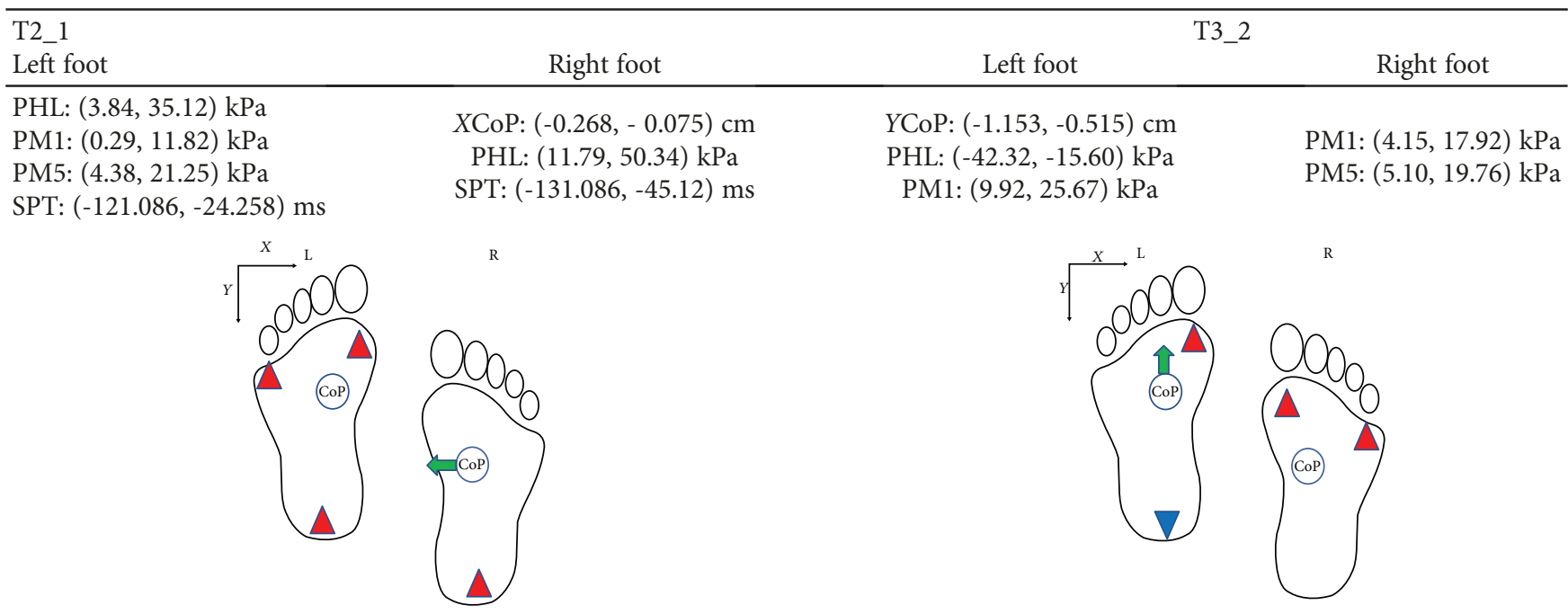

Initials are defined in Table 1. Red triangles and blue inverted triangles show pressure increases and decreases, respectively. Arrows show CoP shifts.

TABLE 7: Study B: gait parameter changes categorized by perceived LBP throughout pregnancy.

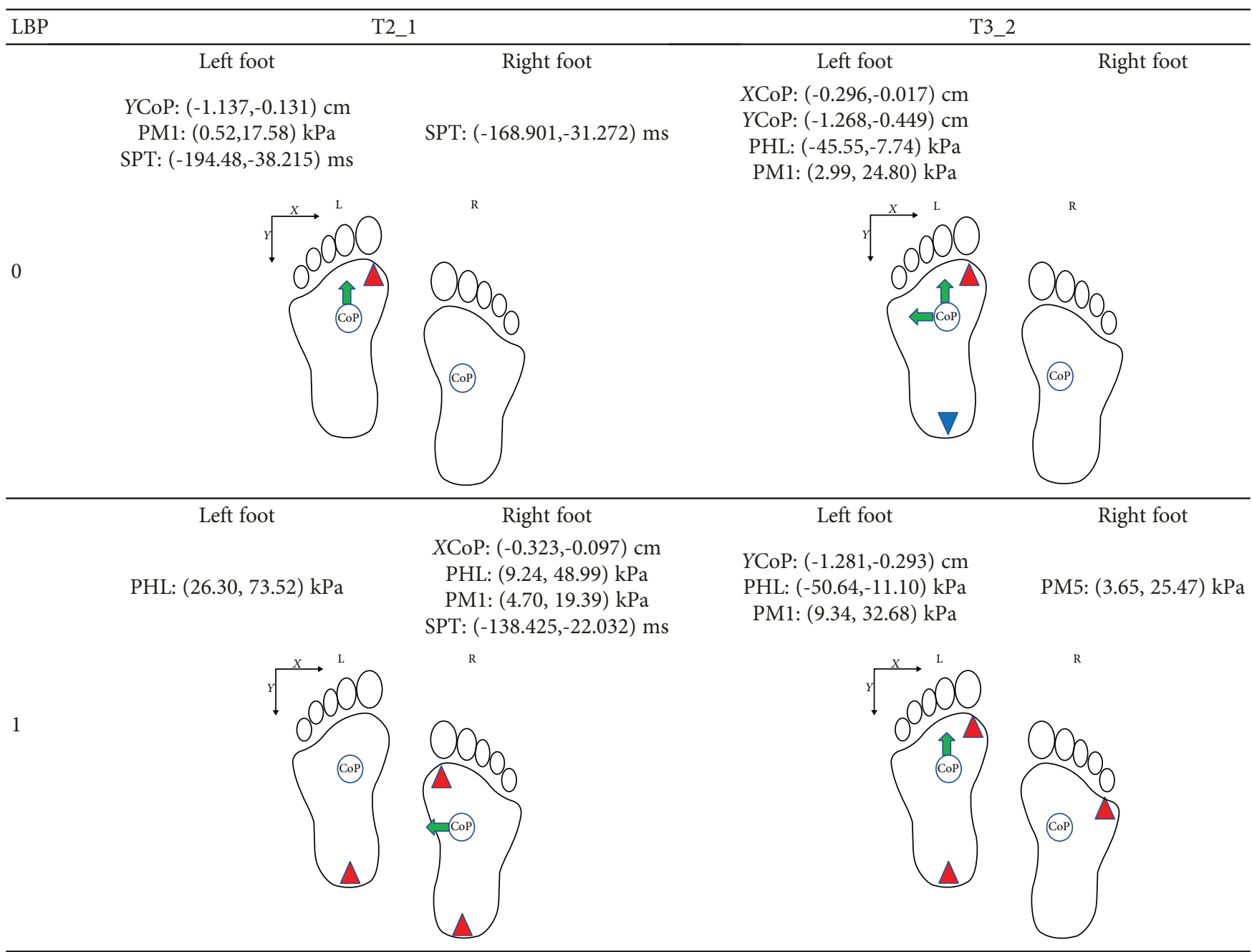


TABLE 8: Study C: gait parameter changes categorized by perceived LBP transitions between consecutive trimesters.

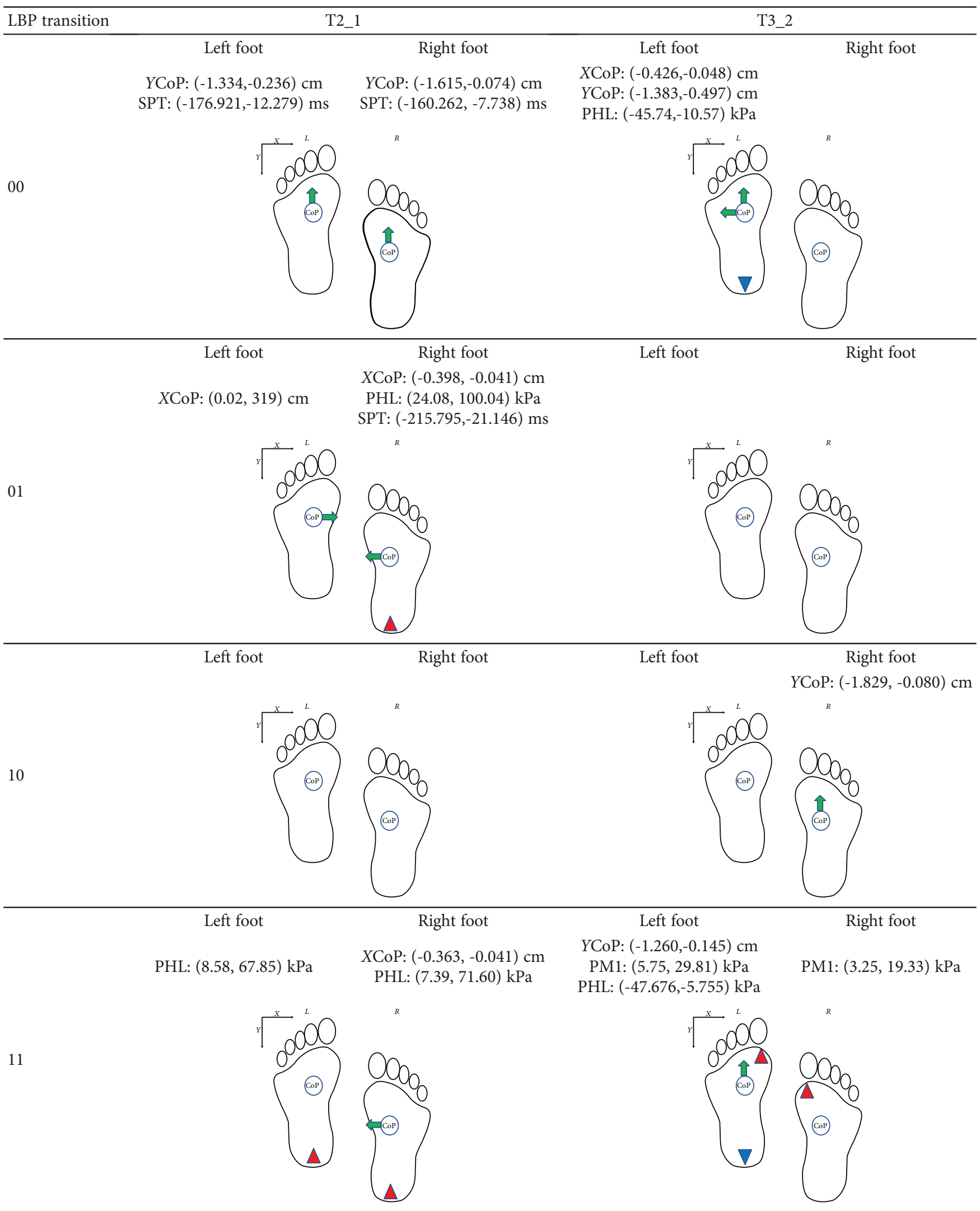

Initials are defined in Table 1. Red triangles and blue inverted triangles show pressure increases and decreases, respectively. Arrows show CoP shifts. 
integrated $\mathrm{CoP}$ tendencies noted. An increase in the first metatarsal pressure $(\mathrm{PM} 1>0)$ can be linked to a pronation tendency throughout pregnancy, which is believed to be a shock-absorption strategy for gaining stability and safety during foot landing [55]. Moreover, a reversible peak pressure trend was observed at the heel, first with a pressure increase in T2 followed by a decrease in T3. Thus, a redistribution of the plantar pressures was noted to the forefoot mainly in T3. This result is in disagreement with some previous reports $[20,21]$, but is in agreement with other studies $[13,14,22,23]$. Pressure increases can obviously be attributed to weight gain and changes in center of gravity/mass, whereas a possible explanation for the pressure redistribution and change of trend at the heel may be related to morphological changes in the foot: increase in foot width and drop in the longitudinal arch height, adopting a pronated posture $[23,30,48,52]$. On the other hand, if the LPB factor is included (Tables 7 and 8), a clear correlation between pain occurrence [1], new pain perception (01), and continued pain (11), with high increases in PHL alone or together with PM1 (forefoot pronation trend), were observed in $\mathrm{T} 2$.

Our SPT results show a significant decrease in this temporal feature between $\mathrm{T} 1$ and $\mathrm{T} 2$ and remains unchanged between T2 and T3 [16], but without a significant correlation with LBP in any study. This decrease in stance phase time might be due to the reduction in step length detected in some kinematics studies as pregnancy progresses $[2,15]$. Indeed, a significant slight decrease of the single-support time was measured in women between T3 and one year postpartum [2]. On the other hand, comparable longitudinal studies observed no change in this parameter between the two first trimesters $[17,21]$. However, a redistribution of the stance phase time with an increase in the contact times and pressures in the midfoot and forefoot between the two first trimesters was also reported that may be explained as an adaptive modification of the foot rollover process [17, 22]. This foot rollover modification was consistent with our results of a shifted CoP towards the forefoot and increased forefoot loads in $\mathrm{T} 2$.

Our results show a clear, repeated asymmetric trend between the right and left feet that made it difficult to analyze them jointly. This may explain previous apparently contradictory results from plantar pressure studies during pregnancy. However, in this work, a significant correlation was observed between LBP occurrence and rise with a pronation tendency in both feet around T2 without a shifted $\mathrm{CoP}$ towards the forefoot. As key evidence from this study, according to the LBP rating data, certain anatomical mechanisms for compensation and redistribution mainly take place between T2 and T3 (and are also reported in the literature), allowing pregnant women to adapt their gait to their changing condition with less perceived LBP such as a lateral and anteriorly shifted $\mathrm{CoP}$ with a redistributed forefoot plantar load. To detect and correct these gait changes associated with low back pain by physical or instrument means could lead to a reduction in its incidence and/or morbidity during pregnancy.

\section{Data Availability}

The data used to support the findings of this study are available from the corresponding author upon request.

\section{Conflicts of Interest}

The authors declare no conflicts of interest.

\section{Acknowledgments}

The authors would like to thank Prof. Jesús Florido (deceased) because he inspired this work and planned and begun the clinical assay. The authors also wish to offer special thanks to all volunteer participants. This work was funded by the Junta de Andalucía (Spain) under Project P10-TIC5997. This project was partially supported by the European Regional Development Funds (ERDF).

\section{References}

[1] M. E. Franklin and T. Conner-Kerr, "An analysis of posture and back pain in the first and third trimesters of pregnancy," Journal of Orthopaedic \& Sports Physical Therapy, vol. 28, no. 3, pp. 133-138, 1998.

[2] T. Foti, J. R. Davids, and A. Bagley, "A biomechanical analysis of gait during pregnancy*," The Journal of Bone and Joint Surgery-American Volume, vol. 82, no. 5, pp. 625-632, 2000.

[3] A. Fast, D. Shapiro, E. J. Ducommun, L. W. Friedmann, T. Bouklas, and Y. Floman, "Low-back pain in pregnancy," Spine, vol. 12, no. 4, pp. 368-371, 1987.

[4] H. C. Ostgaard, G. B. J. Andersson, and K. Karlsson, "Prevalence of back pain in pregnancy," Spine, vol. 16, no. 5, pp. 549-552, 1991.

[5] L. Norén, S. Östgaard, G. Johansson, and H. C. Östgaard, "Lumbar back and posterior pelvic pain during pregnancy: a 3-year follow-up," European Spine Journal, vol. 11, no. 3, pp. 267-271, 2002.

[6] W. H. Wu, O. G. Meijer, K. Uegaki et al., "Pregnancy-related pelvic girdle pain (PPP), I: terminology, clinical presentation, and prevalence," European Spine Journal, vol. 13, no. 7, pp. 575-589, 2004.

[7] S. Sencan, E. E. Ozcan-Eksi, I. Cuce, S. Guzel, and B. Erdem, "Pregnancy-related low back pain in women in Turkey: prevalence and risk factors," Annals of Physical and Rehabilitation Medicine, vol. 61, no. 1, pp. 33-37, 2018.

[8] P. Katonis, A. Kampouroglou, A. Aggelopoulos et al., "Pregnancy-related low back pain," Hippokratia, vol. 15, no. 3, pp. 205-210, 2011.

[9] J. K. Lymbery and W. Gilleard, "The stance phase of walking during late pregnancy: temporospatial and ground reaction force variables," Journal of the American Podiatric Medical Association, vol. 95, no. 3, pp. 247-253, 2005.

[10] F. P. Carpes, D. Griebeler, J. F. Kleinpaul, L. Mann, and C. B. Mota, "Women able-bodied gait kinematics during and post pregnancy period," Revista Brasileira de Biomecânica, vol. 9, pp. 33-40, 2008.

[11] L. Hagan and C. K. Wong, "Gait in pregnant women," Journal of Women's Health Physical Therapy, vol. 34, no. 2, pp. 46-56, 2010. 
[12] R. Sawa, T. Doi, T. Asai, K. Watanabe, T. Taniguchi, and R. Ono, "Differences in trunk control between early and late pregnancy during gait," Gait \& Posture, vol. 42, no. 4, pp. 455-459, 2015.

[13] Q. Mei, Y. Gu, and J. Fernandez, "Alterations of pregnant gait during pregnancy and post-partum," Scientific Reports, vol. 8, no. 1, p. 2217, 2018.

[14] E. Karadag-Saygi, F. Unlu-Ozkan, and A. Basgul, "Plantar pressure and foot pain in the last trimester of pregnancy," Foot \& Ankle International, vol. 31, no. 2, pp. 153-157, 2010.

[15] W. L. Gilleard, "Trunk motion and gait characteristics of pregnant women when walking: report of a longitudinal study with a control group," BMC Pregnancy and Childbirth, vol. 13, no. 1, 2013.

[16] M. Branco, R. Santos-Rocha, L. Aguiar, F. Vieira, and A. Veloso, "Kinematic analysis of gait in the second and third trimesters of pregnancy," Journal of Pregnancy, vol. 2013, Article ID 718095, 9 pages, 2013.

[17] Y. Zhang, H. Lu, Y. Gu, and N. Hu, "Characteristics of the centre of pressure progression for pregnant women during walking," International Journal of Biomedical Engineering and Technology, vol. 17, no. 4, pp. 387-397, 2015.

[18] M. C. Lonergan, E. J. Severin, B. J. Doleman, S. A. Beaber, R. H. Grubbs, and N. S. Lewis, "Array-based vapor sensing using chemically sensitive, carbon black-polymer resistors," Chemistry of Materials, vol. 8, no. 9, pp. 2298-2312, 1996.

[19] J. W. Błaszczyk, A. Opala-Berdzik, and M. Plewa, “Adaptive changes in spatiotemporal gait characteristics in women during pregnancy," Gait \& Posture, vol. 43, pp. 160-164, 2016.

[20] M. Nyska, D. Sofer, A. Porat, C. B. Howard, A. Levi, and I. Meizner, "Planter foot pressures in pregnant women," Israel Journal of Medical Sciences, vol. 33, no. 2, pp. 139146, 1997.

[21] J. Goldberg, M. P. Besser, and L. Selby-Silverstein, "Changes in foot function throughout pregnancy," Obstetrics and Gynecology, vol. 97, no. 5, p. S39, 2001.

[22] A. P. Ribeiro, F. Trombini-Souza, I. de Camargo Neves Sacco, R. Ruano, M. Zugaib, and S. M. A. João, "Changes in the plantar pressure distribution during gait throughout gestation," Journal of the American Podiatric Medical Association, vol. 101, no. 5, pp. 415-423, 2011.

[23] J. Mitternacht, A. Klement, and R. Lampe, "Plantar pressure distribution during and after pregnancy," European Orthopaedics and Traumatology, vol. 4, no. 4, pp. 229-236, 2013.

[24] Z. Krkeljas, "Changes in gait and posture as factors of dynamic stability during walking in pregnancy," Human Movement Science, vol. 58, pp. 315-320, 2018.

[25] T. Varol, A. Göker, E. Cezayırlı, S. Özgür, and A. Tuç Yücel, "Relation between foot pain and plantar pressure in pregnancy," Turkish Journal of Medical Sciences, vol. 47, no. 4, pp. 1104-1108, 2017.

[26] A. P. Ribeiro, S. M. A. Joao, and I. C. N. Sacco, "Static and dynamic biomechanical adaptations of the lower limbs and gait pattern changes during pregnancy," Women's Health, vol. 9, no. 1, pp. 99-108, 2013.

[27] D. Casagrande, Z. Gugala, S. M. Clark, and R. W. Lindsey, "Low back pain and pelvic girdle pain in pregnancy," The Journal of the American Academy of Orthopaedic Surgeons, vol. 23, no. 9, pp. 539-549, 2015.
[28] C. Gaymer, H. Whalley, J. Achten, M. Vatish, and M. L. Costa, "Midfoot plantar pressure significantly increases during late gestation," The Foot, vol. 19, no. 2, pp. 114-116, 2009.

[29] J. E. Bullock, G. A. Jull, and M. I. Bullock, "The relationship of low back pain to postural changes during pregnancy," The Australian Journal of Physiotherapy, vol. 33, no. 1, pp. 10-17, 1987.

[30] F. J. Vico Pardo, A. López del Amo, M. Pardo Rios, G. Gijon-Nogueron, and C. C. Yuste, "Changes in foot posture during pregnancy and their relation with musculoskeletal pain: a longitudinal cohort study," Women and Birth, vol. 31, no. 2, pp. e84-e88, 2018.

[31] S. D. Liddle and V. Pennick, "Interventions for preventing and treating low-back and pelvic pain during pregnancy," Cochrane Database of Systematic Reviews, no. 9, article CD001139, 2015.

[32] J. F. Clapp III, "Exercise during pregnancy," Clinics in Sports Medicine, vol. 19, no. 2, pp. 273-286, 2000.

[33] A. H. A. Razak, A. Zayegh, R. K. Begg, and Y. Wahab, "Foot plantar pressure measurement system: a review," Sensors, vol. 12, no. 7, pp. 9884-9912, 2012.

[34] H. Zhu, N. Maalej, J. G. Webster, W. J. Tompkins, P. Bach-Y-Rita, and J. J. Wertsch, "An umbilical dataacquisition system for measuring pressures between the foot and shoe," IEEE Transactions on Biomedical Engineering, vol. 37, no. 9, pp. 908-911, 1990.

[35] T. J. Salpavaara, J. A. Verho, J. O. Lekkala, and J. E. Halttunen, "Embedded capacitive sensor system for hip surgery rehabilitation: online measurements and long-term stability," in 2008 30th Annual International Conference of the IEEE Engineering in Medicine and Biology Society, Vancouver, BC, Canada, August 2008.

[36] L. Shu, T. Hua, Y. Wang, Q. Qiao Li, D. D. Feng, and X. Tao, "In-shoe plantar pressure measurement and analysis system based on fabric pressure sensing array," IEEE Transactions on Information Technology in Biomedicine, vol. 14, no. 3, pp. 767-775, 2010 .

[37] D. Vilarinho, A. Theodosiou, C. Leitão et al., "POFBG-embedded cork insole for plantar pressure monitoring," Sensors, vol. 17, no. 12, 2017.

[38] F. Martinez-Marti et al., "A preliminary study of the relation between back-pain and plantar-pressure evolution during pregnancy," in 2015 37th Annual International Conference of the IEEE Engineering in Medicine and Biology Society (EMBC), Milan, Italy, August 2015.

[39] F. Martínez-Martí, M. S. Martínez-García, S. G. García-Díaz, J. García-Jiménez, A. J. Palma, and M. A. Carvajal, "Embedded sensor insole for wireless measurement of gait parameters," Australasian Physical \& Engineering Sciences in Medicine, vol. 37, no. 1, pp. 25-35, 2014.

[40] F. Martínez-Martí, J. L. González-Montesinos, D. P. Morales et al., "Validation of instrumented insoles for measuring height in vertical jump," International Journal of Sports Medicine, vol. 37, no. 5, pp. 374-381, 2016.

[41] A. Williamson and B. Hoggart, "Pain: a review of three commonly used pain rating scales," Journal of Clinical Nursing, vol. 14, no. 7, pp. 798-804, 2005.

[42] M. Muñoz-Organero, C. Littlewood, J. Parker, L. Powell, C. Grindell, and S. Mawson, "Identification of walking strategies of people with osteoarthritis of the knee using insole pressure sensors," IEEE Sensors Journal, vol. 17, no. 12, pp. 3909-3920, 2017. 
[43] P. Porwik, J. Zygula, R. Doroz, and R. Proksa, "Biometric recognition system based on the motion of the human body gravity centre analysis," Journal of Medical Informatics \& Technologies, vol. 15, pp. 61-70, 2010.

[44] I. M. Mogren and A. I. Pohjanen, "Low back pain and pelvic pain during pregnancy: prevalence and risk factors," Spine, vol. 30, no. 8, pp. 983-991, 2005.

[45] A. Gutke, H. C. Östgaard, and B. Öberg, "Predicting persistent pregnancy-related low back pain," Spine, vol. 33, no. 12, pp. E386-E393, 2008.

[46] J. W. George, C. D. Skaggs, P. A. Thompson, D. M. Nelson, J. A. Gavard, and G. A. Gross, "A randomized controlled trial comparing a multimodal intervention and standard obstetrics care for low back and pelvic pain in pregnancy," American Journal of Obstetrics and Gynecology, vol. 208, no. 4, pp. 295.e1-295.e7, 2013.

[47] R. A. Block, L. A. Hess, E. V. Timpano, and C. Serlo, "Physiologic changes in the foot during pregnancy," Journal of the American Podiatric Medical Association, vol. 75, no. 6, pp. 297-299, 1985.

[48] P. Ramachandra, P. Kumar, A. Kamath, and A. G. Maiya, "Do structural changes of the foot influence plantar pressure patterns during various stages of pregnancy and postpartum?," Foot \& Ankle Specialist, vol. 10, no. 6, pp. 513-519, 2017.

[49] C. Lang and T. Hubert, "A colour ripeness indicator for apples," Food and Bioprocess Technology, vol. 5, no. 8, pp. 3244-3249, 2012.

[50] S. Ribas and E. Guirro, "Análise da pressão plantar e do equilíbrio postural em diferentes fases da gestação," Revista Brasileira de Fisioterapia, vol. 11, no. 5, pp. 391-396, 2007.

[51] R. Alvarez, I. A. Stokes, D. E. Asprinio, S. Trevino, and T. Braun, "Dimensional changes of the feet in pregnancy," The Journal of Bone \& Joint Surgery, vol. 70, no. 2, pp. 271274, 1988.

[52] N. A. Segal, E. R. Boyer, P. Teran-Yengle, N. A. Glass, H. J. Hillstrom, and H. J. Yack, "Pregnancy leads to lasting changes in foot structure," American Journal of Physical Medicine \& Rehabilitation, vol. 92, no. 3, pp. 232-240, 2013.

[53] J. A. Coplan, "Rotational motion of the knee: a comparison of normal and pronating subjects," The Journal of Orthopaedic and Sports Physical Therapy, vol. 10, no. 9, pp. 366-369, 1989.

[54] J. Borg-Stein and S. A. Dugan, "Musculoskeletal disorders of pregnancy, delivery and postpartum," Physical Medicine and Rehabilitation Clinics of North America, vol. 18, no. 3, pp. 459-476, 2007.

[55] P. Ponnapula and J. S. Boberg, "Lower extremity changes experienced during pregnancy," The Journal of Foot and Ankle Surgery, vol. 49, no. 5, pp. 452-458, 2010. 


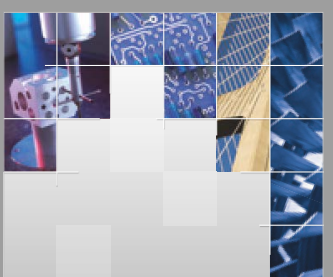

\section{Enfincering}
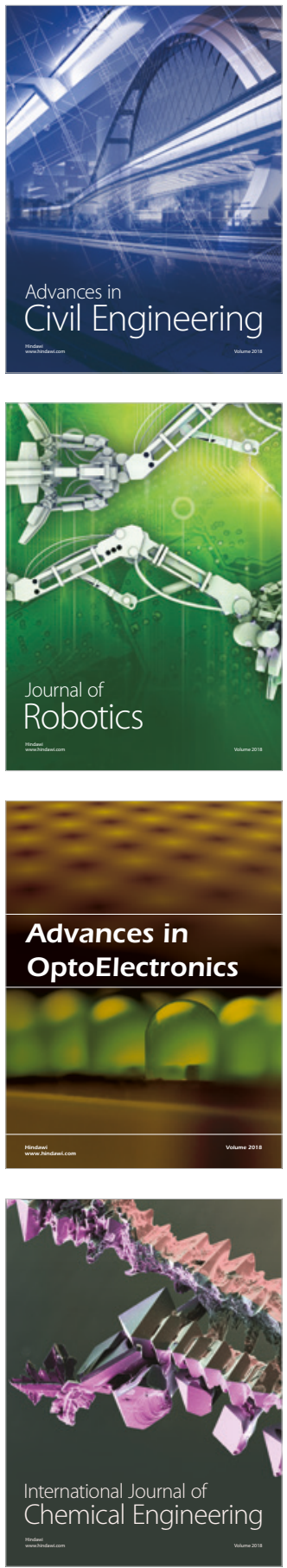

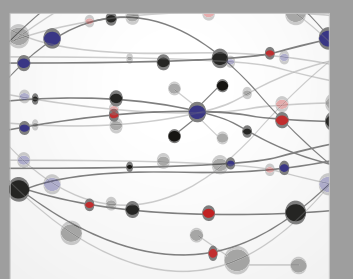

\section{Rotating \\ Machinery}

The Scientific World Journal

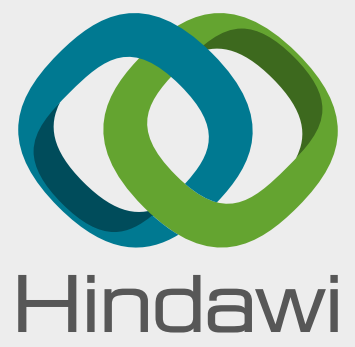

Submit your manuscripts at

www.hindawi.com
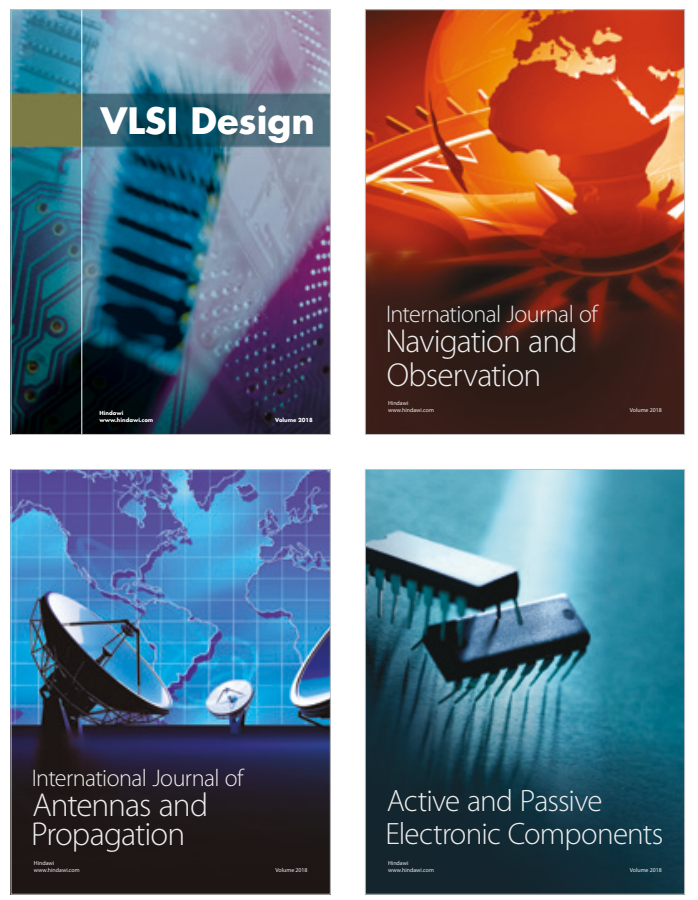
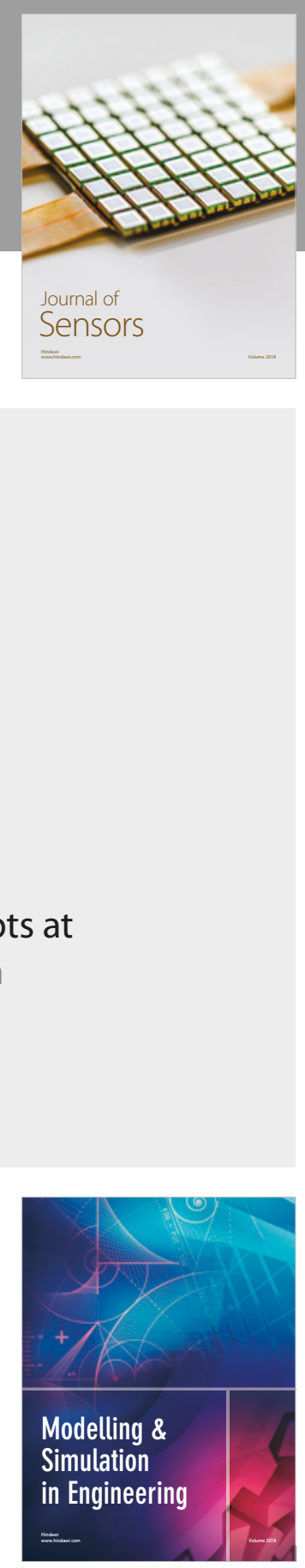

\section{Advances \\ Multimedia}
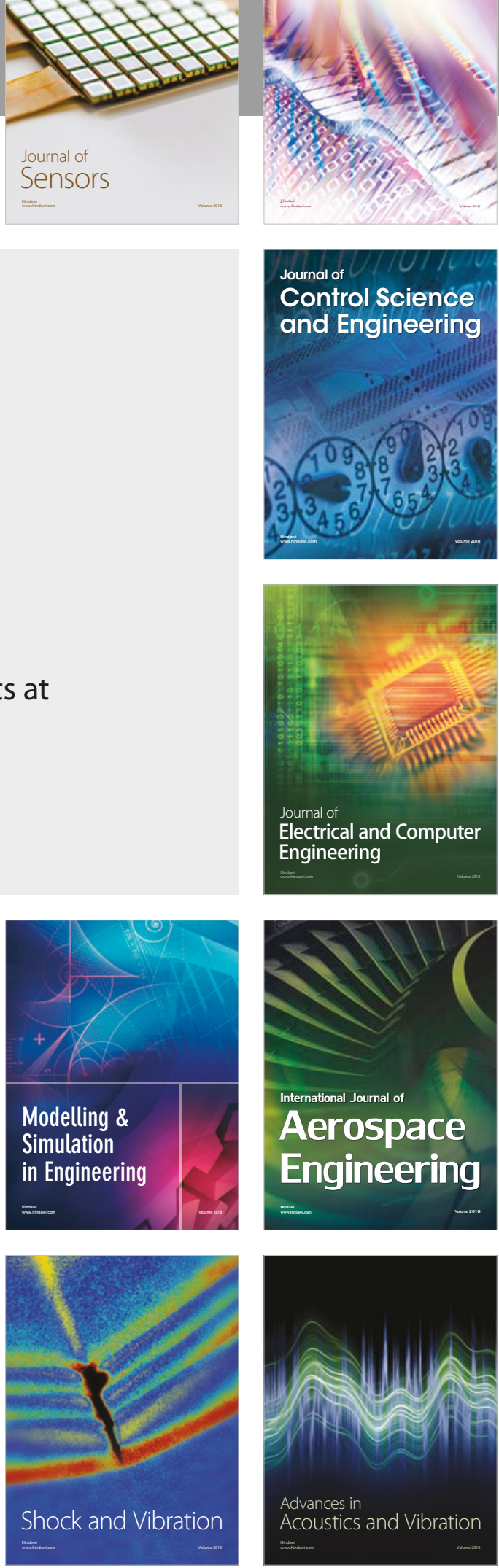